\title{
The Study of Strange Nuclei
}

\author{
R.E. Chrien
}

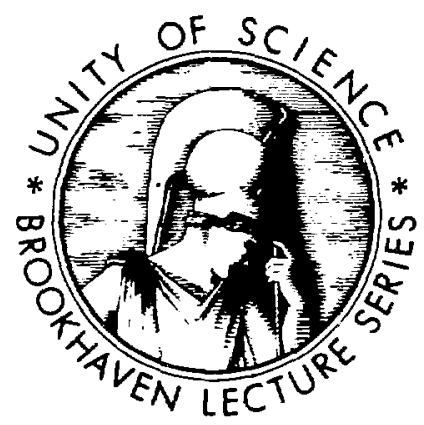

DISCLAIMER

This report was prepared as an acculnt of work sponsored by an agency of the United States Government. Neither the United States Government nor any agency thereof, nor any of their employees, makes any warranty, express or implied, or assumes any legal liability or responsibility for the accuracy. completeness, or usefulness of any information, apparatus, product, or prosess disclosed, or represents that its use would not infringe privately owned rights. Reference herein to any specific commercial product, process, or service by trade name, trademark, manufacturer, or otherwise does not necessarily constitute or imply its endorsement, recommendation, or favoring by the United States Government or any agency thereof. The views and opinions of authors expressed herein do not necessarily state or reflect those of the United States Government or any agency thereof.

\section{BROOKHAVEN LECTURE SERIES}

Number 195, December 8, 1982 


\section{DISCI IMFR}

This report was prepared as an account of work sponsored by an agency of the United States Government. Neither the United States Government nor any agency thereof, nor any of their employees, nor any of their contractors, subcontractors, or their employees. makes any warranty, express or implied, or assumes any legal liability or responsibility for the accuracy, completeness, or usefulness of any information. apparatus, product, or process disclosed, or represents that its use would not infringe privately owned rights. Reference herein ${ }^{+} \mathrm{r}$ any specific commercial product, process, or service by trade name, trademark, man 4 acturer, or otherwise, does not necessarily constitute or imply its endorsement, recommendation, or favoring by the United States Government or any agency, contractor or subcontractor thereof. The views and -opinions of authors expressed herein do not necessarily state or reflect th. use of the United States Government or any agency, contractor or subcontractor thereof.

\section{Printed in the United States of America}

Available from

National Technical Information Service

U.S. Department of Commerce

5285 Port Royal Road

Springfield, VA 22161

NTIS price codes:

Printed Copy: A02; Microfiche Copy: A01 


\section{FOREWORD}

The Brookhaven Lectures, held by and for the Brookhaven staff, are meant to provide an intellectual meeting ground for all scientists of the Laboratory. In this role they serve a double purpose: they are to acquaint the listeners with new developments and ideas not only in their own field, but also in other important fields of science, and to give them a heightened awareness of the aims and potentialities of Brookhaven National Laboratory.

Before describing some recent research or the novel design and possible uses of a machine or apparatus, the lecturers attempt to familiarize the audience with the background of the topic to be treated and to define unfamiliar terms as far as possible.

Of course we are fully conscious of the numerous hurdles and pitfalls which necessarily beset such a venture. In particular, the difference in outlook and meihod between physical and biological sciences presents formidable difficulties. Hov'ever, if we wish to be aware of progress in other fields of science, we have to consider each obstacle as a challenge which ran be met.

The lectures are found to yield some incidental rewards which heighten their spell: In order to organize his taik the lecturer has to look at his work with a new, wider perspective, which provides a satisfying contrast to the often very specializad point of view from which he usually approaches his theoretical or experimental research. Conversely, during the discussion period after his talk, he may derive valuable stimulation irom searching questions or technical advice received from lisieners with different scientific backgrounds. The audience, on the other hand, has an opportunity to see a colleague who may have long been a friend or acquaintance in a new and interesting light.

Gertrude Scharff-Goldhaber

The drawing on the cover is taken from a 5th Century B.C. relief on the Acropolis in Athens, the "Dreaming Athena," by an unknown sculptor. 
INTRODUCTION OF ROBERT CHRIEN

BROOKHAVEN LECTURE SPEAKER

by Maurice GoIdhaber

Lecember 8, 1982

This year the world has been commemorating the discovery of the neutron made fifty years $9 g 0$. Twenty-five years after it was discovered, a young man came here who has since made many very important contributions to our understanding of interactions of neutrons with nuclei. This was Bob Chrien, who joined the Physics Department at Brookhaven in June of 1957 as a Postdoctoral Fellow. He subsequently became a staff member, and at present he holds the title of Senior Physicist and is the leader of the Intermediate Energy Nuclear Physics Group.

Bob received his undergraduate training at Rensselaer Polytechnic Institute, and he holds a Ph.D. degree from Case Institute, now known as Case Western Reserve University. He did his work on something familiar to me-photonuclear reactions. At Brookhaven he joined the group of the late Donald J. Hughes and becane a close co-worker. Together with Harry Palevsky he founded the intermediate energy program at Brookhaven.

For many years he was the group leader of the Neutron Nuclear Physics Group. He has worked on the measurement of neutron cross sections and resonance neutron capture gamma rays, and he has filled many volumes with important and vital data. When the High Flux Beam Reactor became operational, the Neutron Nuclear Group was the first one to take data there and to publish results. This is typical of Bob. He is immediately there when there is a chance to do interesting research.

In addition to being here, Bob spent two-and-one-half years as the Brookhaven representative at Chalk River and joined in their research program. He has also carried out experiments at the Los Alamos Meson Facility.

In 1980 he assumed the leadership of the Intermediate Energy Group and has since directed the program which he will describe tonight.

Bob Chrien is a member and past chairman of both the DOE Nuclear Data Committee and the Nuclear Energy Agency Nuclear Data Committee. He is a Fellow of the American Physical Society, a member of Sigma Xi, the AAAS, the New York Academy of Sciences, the Nuclear Physics Division of the American Chemical Society, and the author of a number of books, some written by himself, some edited by him. I need not say that he has innumerable articles in the research literature.

We are all looking forward to hearing from Bob about strange nuclei. 
THE STUDY OF STRANGE NUCLEI

Introduction

The "frontier" of phystes is not a narrowly localized place. There are frontiers in eiementary particle physics, in hadronic and leptonic forces, in solid state physics, and in nuclear physics. What constitutes a "frontier" is largely a matter of taste--a subjective judgment--and many will disagree on just what or where it is. One definition of a frontier area, however, is embodied in the question: "Will a single experiment, or several related experiments, make a significant difference in our understanding of the field?" If such decisive experiments exist, they are probably in a frontier area.

In the judgment of $V$. F. Welsskopf, as expressed at the Kingston conference more than 20 years ago, much of nuclear physics is no longer a frontier. ${ }^{1}$ He based his remark on the fact that many of the beautiful theories and models used to explain nuclei do not require much fundamental input. "If phenomena can be explained with such little knowledge it means that these phenomena and their explanations are, in some sense, trivial." If, however, nuclear properties such as the positions of energy levels and their spins and parities can be related to the properties of the forces between elementary particles, then the nucleus itself may be considered a laboratory within whose walls these forces may be investigated. The study of hypernuclei is one such area: in hypernuclear research an attempt is made to relate measured nuclear properties to che fundamental forces between baryons.

What is a "hypernucleus"? How does it differ from an ordinary nucleus, and what can be zearned from a comparison between hypernuclei and ordinary nuclei?

Ordinary nuclei are to be thought of as systems of nucleons--particles which can exist in two roughly equivalent states as "protons" and "neutrons." At the level of the strong interaction (that is, the interaction between nucleons), these particles are truly equivalent; they differ only in their electromagnetic interactions, by virtue of the fact that the proton carries a positive electrical charge.

Developments over the past two decades in elementary particle physics have shown that nucleons possess an internal structure: they are made up of "quarks" bound together by "gluons." This picture provides a unified concept for all hadrons, or strongly interacting particles. These can be thought of as baryons (made up of 3 quarks) and mesons (made of quark-antiquark pairs). ordinary nucleons (protons and neutrons) are considered to be made of "up" and "down" quarks. It is an open question at present whether this internal structure has any implications for nuclear physics--there are no sinple aspects of the many-body nuclear structure problems which would be affected at low excitation energies; at higher energies there unght be.

There is a domain of nuclear physics, however, where the internal structure is decisive. To understand this domain, one must go back to cosmic-ray experiments of the late 40s and early 50s, in which particles with unusual properties were produced in high energy collisions between $\pi$ mesons and protons. These particles were always produced in pairs, one member being heavier than an ordinary nucleon, and the other lighter. The heavier one became known as a "hyperon," a member, along with nucleons, of the class of heavy particles called baryons. The baryons interact with each other via strong forces carried by intermediaries called mesons, as first suggested by Yukawa in 1935. A diagram of hyperon production is shown in Figure 1 as an example of the reaction 


$$
\pi^{-}+p+\Lambda+k^{0}
$$

where the neutral particles subsequently decay

$$
\begin{aligned}
\Lambda & +p+\pi^{-}, \\
K^{0} & \rightarrow \pi^{+}+\pi^{-} .
\end{aligned}
$$

The fact that these unusual or "strange" new particles were always produced in pairs (termed "associated" production by A. Pais) suggested a new quantum number and selection rule for elementary particle physics. Gell-Mann and Nishijima proposed the quancum number called "strangeness," S, and postulated that $S$ is conserved in all strong interactions. Thus the $\Lambda$ was assigned $S=-1$, and the $K^{0}, S=+1$. Ordinary nucleons (protons and neutrons) as well as the familar $\pi$ meson, the mediator of the strung force, were assigned $S=0$. Associated production is therefore nothing more than the expression of the rule

$$
\begin{gathered}
\pi^{-}+p+\Lambda+k^{0} \\
0+0=-1+1 .
\end{gathered}
$$

The subsequent decays of the $k^{0}$ and $\Lambda$ violate strangeness conservation; they are "weak" decays and are similar to the weak decay of ordinary nucleons, such as beta decay. Thus the $\Lambda$ is a long-lived particle, at least on a nuclear scale,

$$
\begin{aligned}
\Lambda & +p+\pi^{-}, \\
-1 & \neq 0+0 ; \quad T_{1 / 2}=200 \times 10^{-12} \mathrm{sec} .
\end{aligned}
$$

This is an important point. The $200 \times 10^{-12} \mathrm{sec}$ is to be compared with the lifetime of a nuclear state. For example, a nuclear excitation at an excitation energy of $\approx 7$ to $8 \mathrm{MeV}$ has a decay width of the order of $1 \mathrm{eV}$, and by the uncertainty principle

$\Delta E \Delta t \approx \hbar \approx 6.6 \times 10^{-16} \mathrm{eV}-\mathrm{sec}$,

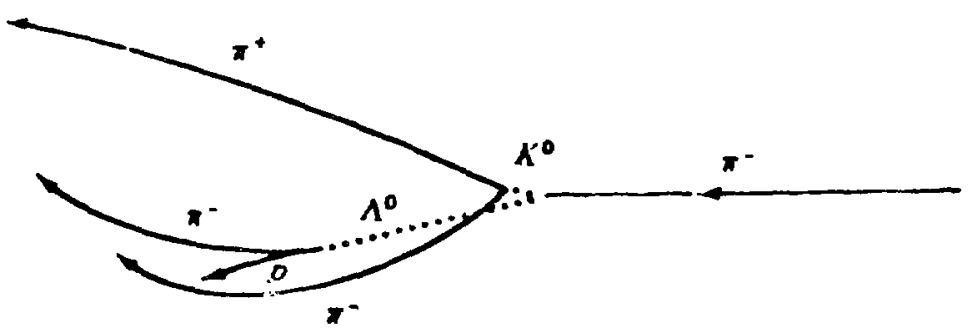

Figure 1. Schematic illustration of the reaction $\pi^{-}+p+\Lambda+K^{0}$, as recorded in a nuclear emulsion. 
it has a lifetime, $\Delta t$, of about $10^{-15} \mathrm{sec}$. Thus the $\Lambda$ hyperon lives for about a thousand nuclear lifetimes.

The relevance of this is as follows. Copious beams of $\mathrm{K}^{-}$mesons are avallable from a high energy accelerator such as the Brookhaven Alternating Gradient Synchrotron (AGS). Suppose a target of nuclei is placed in the beam, and the neutrons in the nuclear target undergo the following reaction:

$$
\mathrm{k}^{-}+\mathrm{n}+\Lambda+\pi^{-} \text {. }
$$

The neutron has been replaced by a $\Lambda$, and it lives long enough to partake of nuclear motions. The resulting nucleus is called a "strange" nucleus, or, as originally suggested by $M$. Goldhaber, a hypernucleus.

It is instructive to compare the quark structure of these strange particles with those of neutrons and protons, as shown in Figure 2. The $\Lambda$ hyperon differs from its analogs in that it has a strange quark. The crucial questions of the study of hypernuclei focus on the following issues. How do nuclear systems containing a strange hyperon differ from ordinary nuclei? What do these differences tell us about the fundamental nuclear force?

Before proceeding with the discussion of strange nuclei, it is useful to summarize the properties of the $\Lambda$ in comparison with those of the neutron, which it replaces:

Charge
Mass
Spin
Isospin
Lifetime (mean life)
Strangeness

Charge

Isospin

Strangeness

\begin{tabular}{ll}
$\Lambda$ & neutron \\
\hline 0 & 0 \\
$1115.6 \mathrm{MeV}$ & 939.57 \\
$1 / 2$ & $1 / 2$ \\
0 & $1 / 2$ \\
$2.6 \times 10^{-10} \mathrm{~s}$ & $925 \mathrm{~s}$ \\
-1 & 0
\end{tabular}

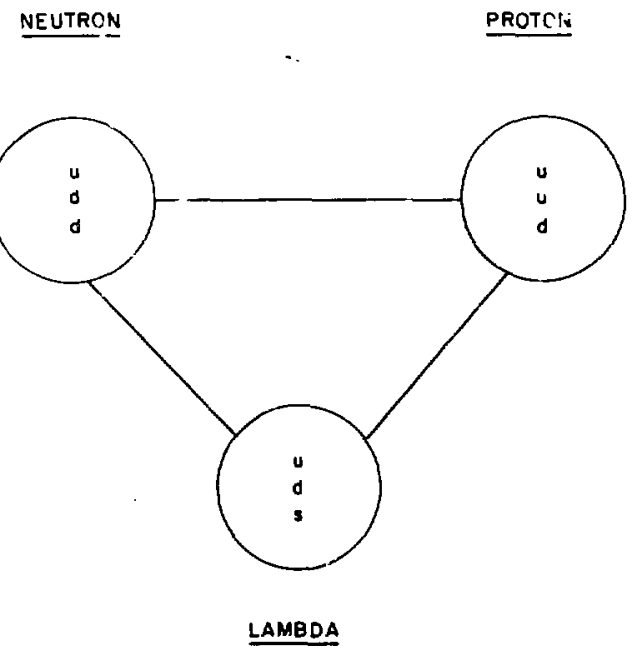

Figure 2. The quark representation of the three lightest baryons: the neutron, proton, and $\Lambda$ hyperon. 
The discovery of a hypernucleus occurred in 1953, when Danysz and Pniewski $^{2}$ reported the observation of the decay process illustrated in Figure 3 in a nuclear emulsion. A high energy particle produced a recoil hypernucleus, which, after slowing down (a process requiring at least $10^{-12} \mathrm{sec}$ ), decayed with the emission of charged pions and nucleons. For about 20 years hypernuclei were studied by means of emulsions or bubble chambers, and they
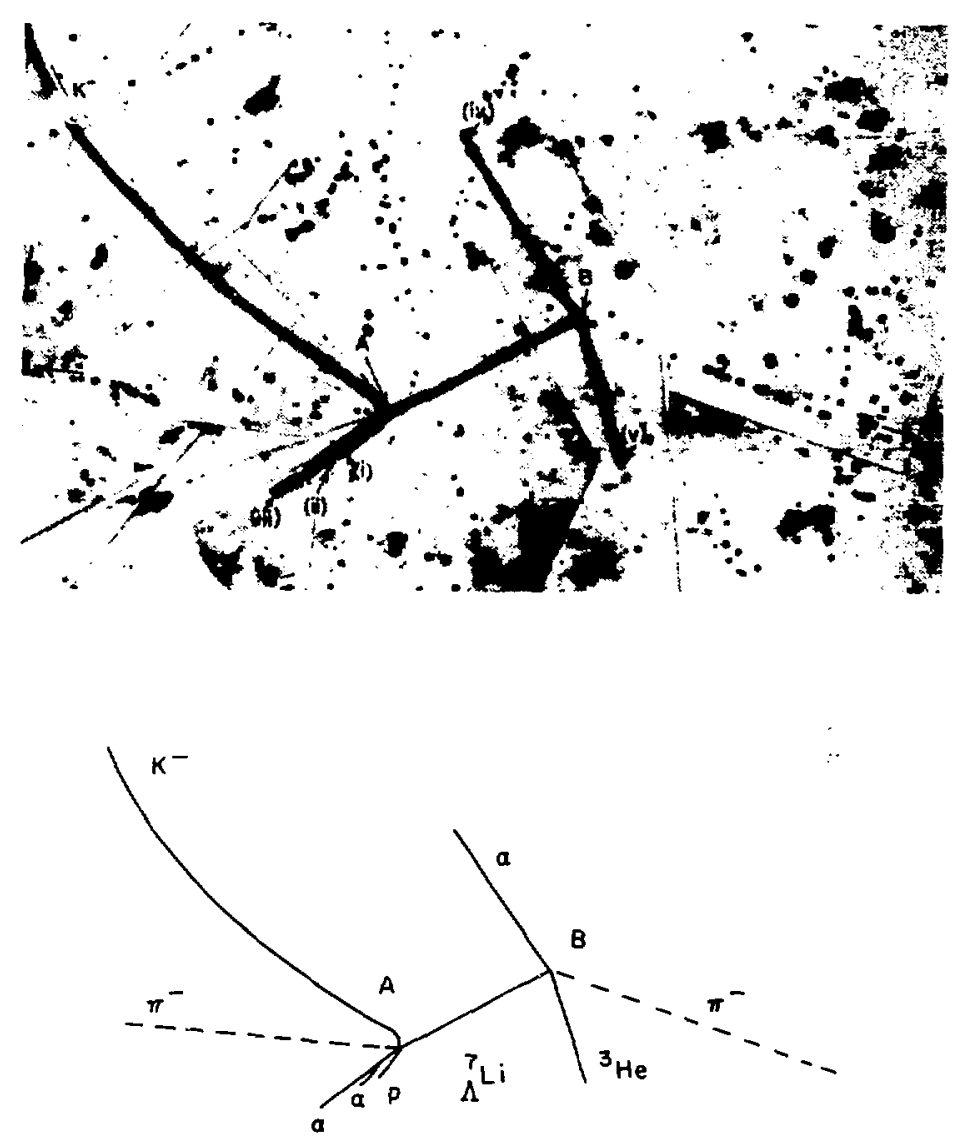

$\longrightarrow 5 \mathrm{O}_{\mu \mathrm{m}} \longrightarrow$

Figure 3. An emulsion photograph illustrating the formation of the hypernucleus $\Lambda^{7} \mathrm{Li}$ : the lower half is a schematic interpretation of the emulsion photograph above. A $K^{-}$interacts with ${ }^{16} \mathrm{O}$ nucleus in the enulsion, producing a $\Lambda^{7} \mathrm{Li}$ and several other charged particles. The $\Lambda^{7}$ Li lives for several hundred picoseconds, and subsequently decays into a pion, ${ }^{3} \mathrm{He}$, and ${ }^{4} \mathrm{He}$ (or $\alpha$ particle) (ref 3 ). 
were identified by the characteristic decay through fragmentacion. From a measurement of the decay products of light nuclei, a consistent plcture of the binding energy of the hypernucleus in its ground state could be obtained. of the two possible decay modes,

$$
\Lambda+N+\pi \quad \text { and } \Lambda+N \rightarrow 2 N \text {, }
$$

the latter is predominant in nuclei and the former in free space. In the second case the mass of the hypernucleus can be obtained from the sum of the decay product masses and energies. Current systematic knowledge oi these separati_n energies is summarized in Figure 4, where they are compared with those for neutrons.

The binding energy shows a smooth dependence on $A$ and contrasts sharply with the neutron binding energy behavior, which looks wildly erratic but is in fact related to the shell structure of the nucleus. This 1llustrates one very important difference between hypernuclei and ordinary nuclei. Since the $\Lambda$ hyper in carries a strangeness quanium number of -1 , it is uninhibited by the Paul $\perp$ principle and may occupy any nuclear orbit, whetier filled with other nucleons or not. Thus in any hypernucleus, the ground state is one in which the $\Lambda$ is found in the lowest, or 1s, shel.1. The $\Lambda$ separation energy reflects only the depth of the nuclear well and does not depend at al.l on the extent of filling of the orbitals by other nucleons.

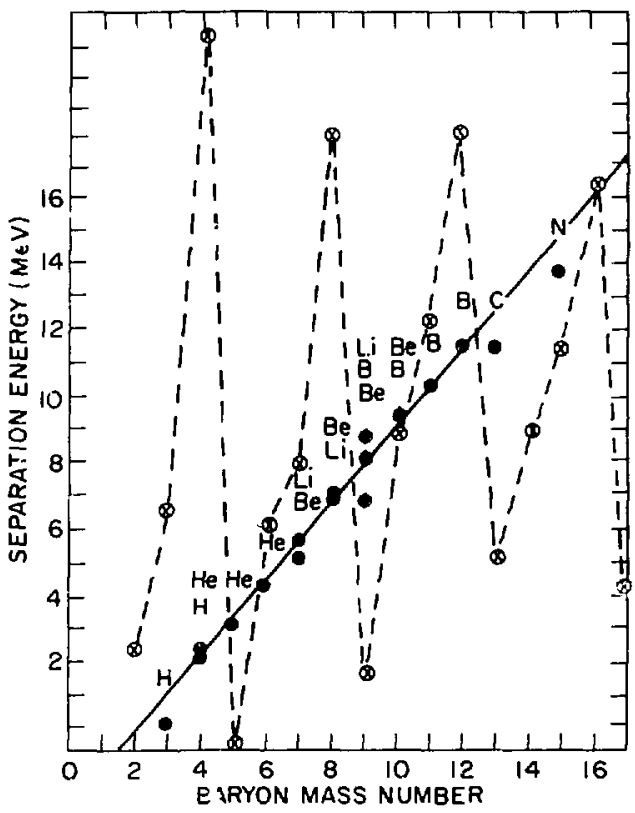

Figure 4. Separation energies of known hypernuclei (points) compared to typical neutron separation energies (open circles) for similar nuclear mass numbers (A). The smooth variation of ground state separation energy for hypernuclei is an indication of the absence of the Pauli principle, which inhibits like particles from occupying the same orbitals. 
A semiquantitative estimate of this separation (or binding) energy can be derived by applying Heisenberg's uncertainty principle to a square well potential. According to this, the kinetic energy of the ground state (gs) $\Lambda$ is

Therefore,

$$
\begin{aligned}
\Delta P & \approx \hbar / R, \\
2 m E & =\hbar^{2} / R^{2}, \\
E & =\hbar^{2} / 2 R^{2}, \\
E_{R} & =V-B_{\Lambda}=\pi^{2} \hbar^{2} / 2 M R^{2}=119 A^{2 / 3} . \\
B_{\Lambda} & =V-119 A^{-2 / 3} .
\end{aligned}
$$

This formula can be fitted with a well depth $\mathrm{V}$ of $30 \mathrm{MeV}$ and, in contrast to the nuclear case, in which a nucleon is added to the lowest unfilled shell, an increasing $B_{\Lambda}$ value with $A$. This result is in reasonable accord with the data from emulsions for light nuclei, but there are no experimental data for heavy nuclei. The value $30 \mathrm{MeV}$ is significantly lower than that for the nuclear case, in which $V \approx 40 \mathrm{MeV}$ for low energy neutrons, and provided the first evidence that the $\Lambda$-nucleon interaction significantly differs from the nucleon-nucleon interaction. Some additional separation energy values have been measured since, but the above information was all that was known about hypernuclef when the state of the field was summarized by Telegdi in 1962.4

\section{Coherent Production of Hypernuclei}

:- the 1960s more intense beams of $K$ mesons became avallable to experimenters so that sophisticated counter experiments could be done to broaden the knowledge of hypernuclei. Almost all of the experimental work in this area has been done at the CERN proton synchrotron and the Brookhaven AGS. The beams produced by these machines make it possible to exploit the characteristics of the reaction mechanism by which the $\Lambda$ is formed:

$$
\begin{gathered}
K^{-}+n \rightarrow \Lambda+\pi^{-} \text {(free), } \\
K^{-}+{ }^{A} Z+{ }_{\Lambda}^{A}+\pi^{-} \text {(niclear). }
\end{gathered}
$$

It is essential, in the reaction above, for the product nucleus $\Lambda^{A} Z$ not to be disrupted by the momentum and energy transferred to it by the $\mathrm{K}^{-}$particle; in other words, the $\Lambda$ must remain a part of the nuclear system. Fortunately, the kinematical properties of this reaction allow a very small momentum transfer:

where

$$
\begin{aligned}
& \mathrm{qT}_{\mathrm{T}} \approx 2|\mathrm{p}| \sin \alpha / 2, \\
& \left|\mathrm{pK}^{-}\right| \approx\left|\mathrm{P}_{\pi}-\right| \simeq|\mathrm{p}| .
\end{aligned}
$$

The situation is depicted schematically in Figure 5, where an incident kaon transforms a neutron to a $\Lambda$, wh the emission of a pion. The recoiling $\Lambda$ carries only a small momentum.

As seen in Figure 6, for kaon beams of 500 to $1000 \mathrm{MeV} / \mathrm{c}$, the recoil momentum of the $\Lambda$ at $0^{\circ}$ is less than $100 \mathrm{MeV} / \mathrm{c}$, which in turn is much less than the $250 \mathrm{MeV} / \mathrm{c}$ typically found in the Fermi motion of the nucleus. Hence there is a high probability that the $\Lambda$ is not ejected and a hypernucleus is 
formed. For Incoming beams of about this momentum, hypernuclei can be produced by just "tickling" the nucleus, leaving its core virtually unchanged.

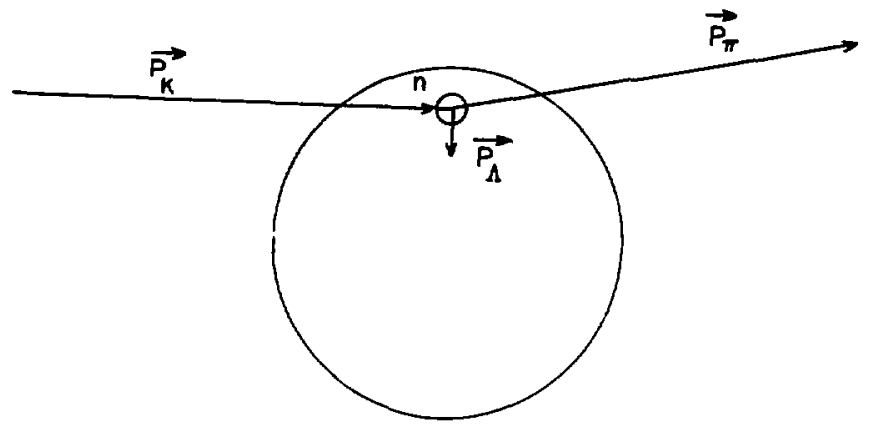

Figure 5. Illustration of the concept of momentum transfer in a nuclear collision involving the $K, \pi$ reaction. When the momentum recoil is small, the chance of producing a coherent nuclear excitation is large.

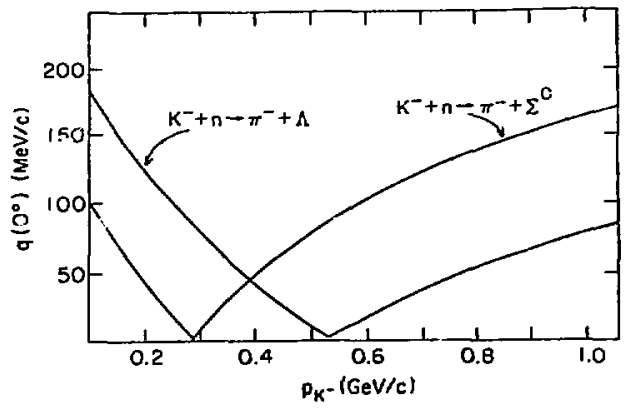

Figure 6. The kinematical dependence of momentum transfer on incident kaon momentum in a $(K, \pi)$ interaction for both $\Lambda$ and $\Sigma$ production.

The Hypernuclear Spectrometer

Kaon-induced reactions are being studied at three facilities: CERN (Geneva), KEK (Japar), and BNL.

The spectroneter at Brookhaven uses the low energy separated beam (LESB-1); this beam is obtained at an angle of about $10^{\circ}$ from the C-1ine production target. The secondary particles (mostly protons, pions, and kaons) pass through magnetic selection elements where negative (or positive) particles of the proper momentun are selected. A beam separator, sometimes termed a wien filter, is used to discriminate between kaons and pions having the sarne monentum. Since kaons (mass $\approx 494 \mathrm{MeV}$ ) are much heavier than pions (miss $\approx 140 \mathrm{MeV}$ ), the pion velocities are higher. The beam separator has crossed electric and magnetic fields; since the ratiu of the electric and magnetic forces on the particles is velocity dependent, it distinguishes between particles of different velocities. The separator discards $90 \%$ of the pions, but the primary pions are so numerous to start with that the output ratio of pions to kaons is 10 to 1 (for negative particles). The pion-induced effects are later discarded in the spectrometer instrumentation.

Because the kaon lifetive is relatively short ( $12.4 \mathrm{~ns})$, the spectrometer must be kept short in length. The kaon beam loss is momentum dependent, being less severe at high momenta because of relativistic time dilation.

Most of the BNL experiments are done at $800 \mathrm{MeV} / \mathrm{c}$. At this momentum the following parameters obtain: $\mathrm{E}^{\mathrm{K}}$ total $=940.23 \mathrm{MeV} ; \quad B_{\mathrm{K}}=0.85085$; $\tau_{\text {eff }}=23.6$ ns; mean 1 ffe distance $=c \beta \tau=6.024$ meters; and half-life distance $=4.2$ meters.

The BNL spectrometer (Figure 7) has two bending magnets between which is placed the target to be studict in a $(K, \pi)$ reaction. These two magnets are used in detarmining the difference between the momenta of the incoming kaon and the outgoing pion. The kaon and pion directions are determined from 


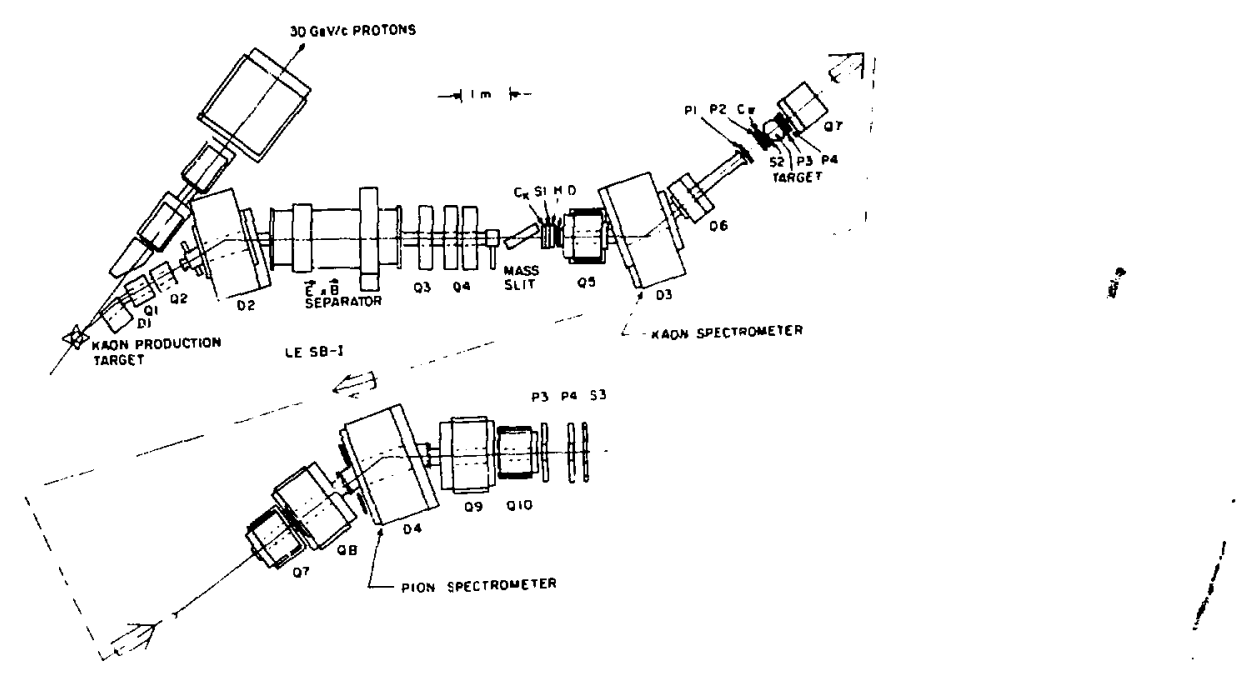

Figure 7. The hypernuclear spectrometer, "Moby Dick," at the the AGS.

position coordinates derived from multiwire proportional counters. The bending of the beatn, which is related to the momentum difference, is also determined from these coordinates. This momentum difference is related to the excitation of the recojiing nucleus in the reaction. The spectrum so obtained is sometimes called a "missing mass spectrum," and it displays the vạrious possible excitation energies of a hypernucleus.

For about $2 \times 10^{12}$ protons per pulse on the production target, about $10^{5}$ kaons are incident on the target for an incident beam acceptance of $+2 \%$. The interval between AGS pulses is 2 to $3 \mathrm{sec}$. The $10^{h}$ pions are rejected by a Cherenkov counter placed at the front of the kaon section of the spectrometer, and they are also rejected by a measurement of the flight time of farticles through the spectrometer as determined by fast scintillators at the front $\left(S_{1}\right)$, at the target $\left(S_{2}\right)$, and at the rear $\left(S_{3}\right)$. At present the resolution available from this spectrometer is about $2 \mathrm{MeV}$ (full width at half maximum), and a solid angle of 12 millisteradian is obtained with a momentum acceptance of $\approx 6 \%$. The spectrometer is mounted on a rotatable platform pivoted at the target position, allowing an angular swing of up to $45^{\circ}$ to the incident kaon beam direction. This capability is unique, and $f$ is important to these studies because it allows one to distinguish between different angular momentum transfers to the nucleus and therefore between different types of nuclear states.

One very important source of background in this experiment arises from the kaon decay process:

$$
\mathrm{K}^{-}+\pi^{-}+\pi^{0}
$$

which occurs in about $21 \%$ of kaon decays. This background is especially troublesome because the $\pi^{-}$from the decay can be confused with the $\pi^{-}$from the nuclear reaction. This background cau be almost totally repressed by reconstructing the kaon and pion trajectories and checking to make sure the event has originated from the target; the decay events are distributed evenly along the beam line. 
The application of the spectrometer to studies of hypernuclei and their energy levels is illustrated by the following discussion of the light nuclei $\Lambda^{12} \mathrm{C}, \Lambda^{13} \mathrm{C}$, and $\Lambda{ }^{16} \mathrm{O}$.

The states of the hypernuclei produced may be described as neutron-hole states of the core coupled to a $\Lambda$. Because of the strong absorption of the $\mathrm{K}^{-}$, the $\mathrm{K}^{-}, \pi^{-}$reaction occurs mostly at the surface, where the valence nucleons are more numerous than are the nucleons of the more strongly bound shells. For example, in ${ }^{12} \mathrm{C}$, the reaction involving the replacement of a $\mathrm{p}_{3 / 2}$ neutron by $a$ is dominant. For a slightly heavier nucleus, for example, ${ }^{16} 0$, a strong contribution is expected from the neutrons in the $p_{1 / 2}$ she11. This kind of reaction is called a "substiturional" reaction, because the $\Lambda$ assumes the same quantum numbers possessed by the neutron it replaces. Because of the freedom from the Pauli principle, the substitutional state is usually not che ground state, however. The ground-state ccafiguration is one in which the $\Lambda$ is always in the lowest $s$ orbit. That orbit is forbidden to additional neutrons, because it is already fully occupied, but not to $\Lambda^{\prime} s$, which are distinguishable from neutrons because of their strangeness.

The degree to which the substitutional state and ground state may be excited in the reaction $\left(\mathrm{K}^{-}, \pi^{-}\right)$depends on the orbital angular monentum transfer, and therefore on the angle at which the pion is emitted with respect to the incident beam. The usefulness of the Moby Dick spectrometer in obtaining angular distributions was demonstrated in the ${ }^{12} \mathrm{C}\left(\mathrm{K}^{-}, \pi^{-}\right)$experiment, the first hypernuclear study at BNL. 6

Figure 8 illustrates formation of the substitutional and ground states in ${ }^{12} \mathrm{C}$. Figure 9 shows an experimental "missing mass" or excitation energy spectrum obtained at a spectrometer angle of $15^{\circ}$. The angular distributions for the two major peaks seen in Figure 9 are plotted in Figure 10. These experimental data serve to provide a detailed picture of the reaction mechanism involved.

$$
{ }^{12} \mathrm{C}\left(\mathrm{K}^{-}, \pi^{-}\right) \text {Experiment }
$$

${ }^{12} \mathrm{C}$ has a filled $\mathrm{p}_{3 / 2}$ shell for both neutrons and protons. The dominant peak in a $\mathrm{K}^{-}, \pi^{-}$reaction at $0^{\circ}$ would be the substitutional peak $\left(\mathrm{p}_{3 / 2} \mathrm{2}^{-1}, \mathrm{p}_{3 / 2}\right)_{\mathrm{n} \Lambda}$. (The notation indicates a neutron vacancy in the $\mathrm{p}_{3 / 2}$ she11 and a $\Lambda$ present in the $\mathrm{p}_{3 / 2}$ she11.) This is evident in Figure 9 near $S_{\Lambda}=0$. There is also evidence for the ground state config"ration $\left(p_{3 / 2^{-1}}, s_{1 / 2}\right)_{n \Lambda}$ at $0^{\circ}$, but at $15^{\circ}$ that peak is much larger. This is consistent with the orbital angular momentum change, $\Delta=1$, required to transform a $\mathrm{P}_{3 / 2}$ into an $s_{1 / 2}$ orbit. The position of the gs peak corresponds to a $\Lambda$ separation energy of $10.79+.11 \mathrm{MeV}$ as compared with two emulsion measurements of $10.76 \pm 0.19$ and $11 . \overline{14} \pm 0.57 \mathrm{MeV}$.

The angular distribution of the gs peak is fully consistent with a $\Delta=1$ multipolarity. The $11-\mathrm{MeV}$ peak is more interesting. First of all it displays the forward peaking at $0^{\circ}$ expected for the $\Delta L=0$ substitutionai reaction. However, the fall-off with angle is not consistent with $\Delta=0$; there is a definite shoulder near $10^{\circ}$. This is not unexpected; a $\mathrm{p}_{3 / 2}$ hole and $\mathrm{p}_{3 / 2}$ particle orbit can couple to give states of $0^{+}$and $2^{+}$, ignoring negative parity states, which is reasonable in this kinematic range.

Furthermore a contribution can be expected from the nearby $\Lambda_{1} 1 / 2$ orbital, particularly if the $\Lambda$ spin-orbit coupling is small. This would result in another $2^{+}$state $\left(\mathrm{p} 3 / 2^{-1}, \mathrm{p} 1 / 2\right)_{\mathrm{n} \Lambda^{*}}$ The $2^{+}$components clearly 


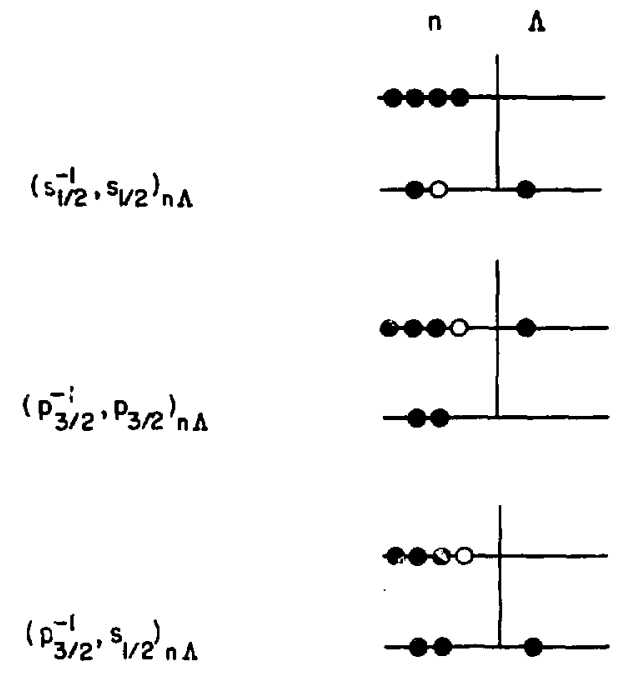

Figure 8. A schematic representation of neutronhole $\Lambda$-particle states in $\Lambda^{12} \mathrm{C}$.

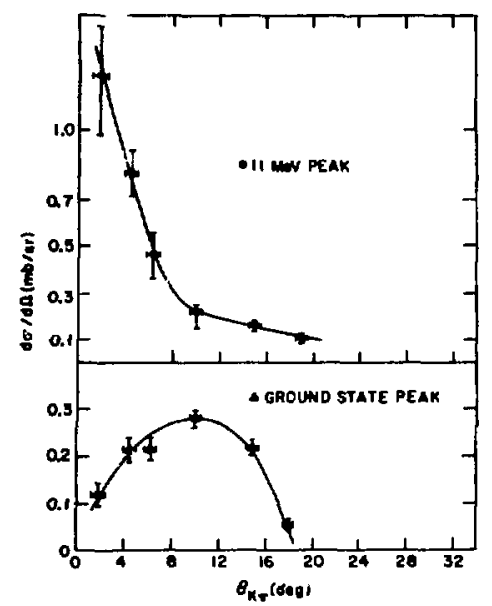

Figure 10. Angular dependence of the ground-state and $11-\mathrm{MeV}$ "peaks" in the $\Lambda^{12} \mathrm{C}$ spectrum. The curve for the latter excltation clearly shows its multiplet character.

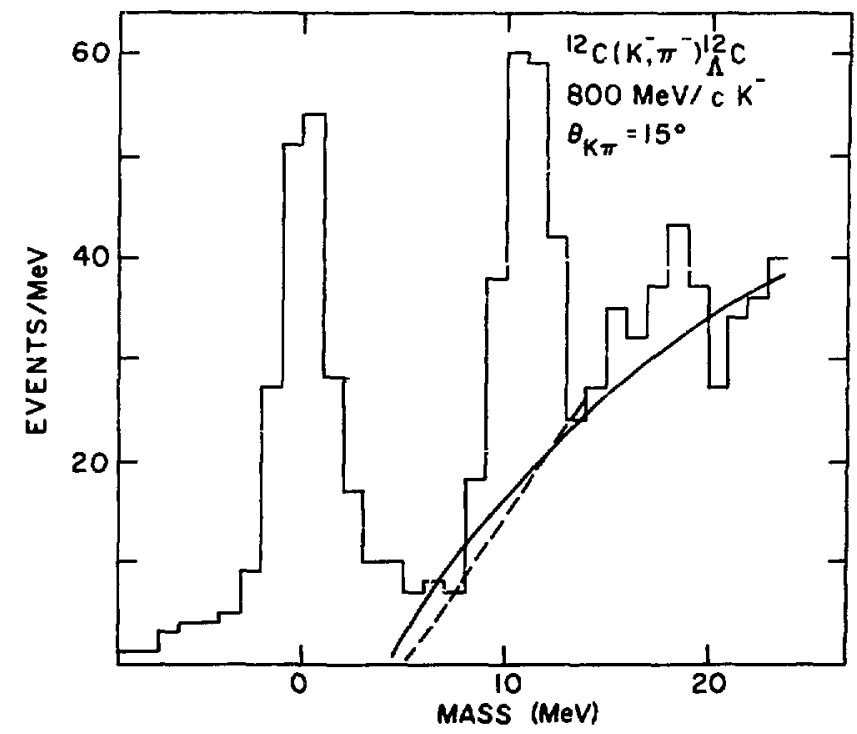

Figure 9. Experimental excitation spectrum for $\mathrm{K}^{-}, \pi^{-}$on ${ }^{12} \mathrm{C}$ to produce $\Lambda^{12} \mathrm{C}$ at $15^{\circ}$. 
affect the angular distribution. These additional $2^{+}$states require orbital angular momentum transfer and are thus evident at large scattering angles.

The finding of only a single $11-\mathrm{MeV}$ peak and no shift in the 11-MeV peak position with angle puts severe restraints on the spin-dependent $\Lambda$-nucleus potential. The splittings allowed are (a) less than $420 \mathrm{keV}$, assuming one $2^{+}$, or (b) less than $820 \mathrm{keV}$, assuming two $2^{+}$states are present, with equal strength at $15^{\circ}$. These numbers should be compared with the 6-MeV splitting between spin-orbit partners $\ell \pm 1 / 2$ in the nuclear case.

Another clue as to the smallness of the nuclear- $\Lambda$ spin-dependent pocentials is obtained from the CERN experiments on $\Lambda^{12} \mathrm{C}$ and $\Lambda^{16} 0$, done at $0^{\circ}$ only. The $\left(\mathrm{K}^{-}, \pi^{-}\right)$spectra obtained at CERN for ${ }^{12} \mathrm{C}$ and ${ }^{16} \mathrm{O}$ at $0^{\circ}$ are shown in Figure 11 , plotted as a function of $\Lambda$-binding energy or, alternatively, transformation energy $M_{H Y}-M_{A}$. This latter quantity is convenient in comparing level structures of different targets.

From the mass-energy relations,

$$
M_{A-1}+M_{N}=M_{A}+S_{N} \text {, }
$$

and

from which

$$
M_{A-1}+M_{\Lambda}=M_{H Y}+s_{\Lambda}
$$

$$
M_{H Y}-M_{A}=\left(M_{\Lambda}-M_{N}\right)+\left(s_{N}-s_{\Lambda}\right)
$$

The quantity $M_{\Lambda}-M_{N}$ is $176 \mathrm{MeV}$, and the quantity $\left(S_{N}-S_{\Lambda}\right)$ is given approximately by the difference in neutron and $\Lambda$ zuclear potentials and is rather insensitive to nuclear size. As seen in Figure 11 , the major peaks are identified as substitutions in the $\mathrm{P} 3 / 2$ shell, and they incur roughly at $M_{H Y}-M_{A} \approx 196 \mathrm{MeV}$. The ground state for ${ }^{12} \mathrm{C}$ consists of the $1 \mathrm{~s}_{1 / 2} \Lambda$ coupled to the $\mathrm{p} 3 / 2$ neutron hole. For ${ }^{16} \mathrm{O}$, just twice as many peaks are seen, since there are contributions from both the $\mathrm{P} 3 / 2$ and $\mathrm{P} 1 / 2$ shells. There are substitutional states $\left(\mathrm{P}_{3 / 2^{-1}}{ }_{1} \cdot \mathrm{P}_{3 / 2}\right)_{\mathrm{n} \Lambda} ;\left(\mathrm{p}_{1 / 2}{ }^{-1}\right.$, $\left.\mathrm{p}_{1 / 2}\right)_{\mathrm{n}}$; and nonsubstitutional states $\left(\mathrm{P}_{3 / 2^{-1}}, \mathrm{~s}_{1 / 2}\right)_{\mathrm{n} \Lambda} ; \quad\left(\mathrm{p}_{1 / 2^{-1}}\right.$, $\left.s_{1 / 2}\right)_{n}$ formed by coupling the $s_{1 / 2} \Lambda$ to the neutron-hole states. The substitutional states are forward peaked, since there is no orbital angular momentum transfer. The $\Lambda s_{1 / 2}$ peaks are weak at forward angles, as expected from the $\Delta \mathrm{L}=1$ transfer from the nucleon.

The energy difference for the $p_{3} / 2^{-}$and $p_{1} / 2^{-h o l e}$ states in $\Lambda^{16}$ is very ${ }_{5}$ nearly equal to the $6.1-\mathrm{MeV}$ difference between the corresponding states in 5 . The presence of the $\Lambda$ seems to have little effect on the spacing. The clear suggestion is that the $\Lambda$-nucleus spin-orbit force is negligible in $\mathrm{p}$-shell nuclei. In fact, calculations by Byoussy, assessing the spin-orbit force as zero, reproduce the energies and intensities of these peaks very well.

A direct measure of the spin-orbit coupling has been given by a recent experiment on $\Lambda^{13} \mathrm{C}$ done at Brookhaven. ${ }^{8}$ This experiment provides a good example of the main features of hypernuclear spectra and it illustrates well the success of a weak-coupling model for hypernuclei. The right side of Figure 12 is a histogram of a $\left(\mathrm{K}^{-}, \pi^{-}\right)$spectrum obtained at $800-\mathrm{MeV} / \mathrm{c}$ kaons and outgoing pions near $0^{\circ}$. This is compared with the positions of known hole states in ${ }^{3} \mathrm{C}$ as determined from the $(p, d)$ pickup reaction. The hypernucleus spectrum is seen to be a superposition of the two spectra formed by coupling an $\mathrm{s}^{-}$or $\mathrm{p}^{-\mathrm{s} h e 11} \Lambda$ with an excited ${ }^{12} \mathrm{C}$ core. The core excitations are those 


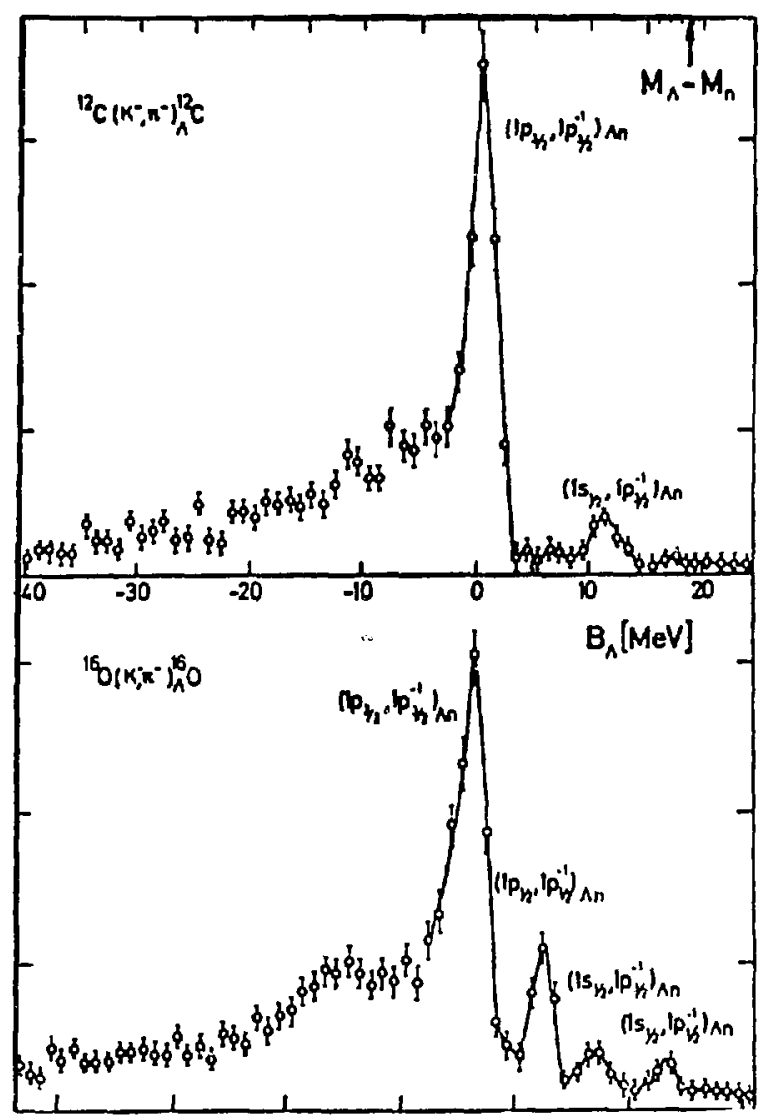

Figure 11. Spectra for $\Lambda^{16} \mathrm{O}$ and $\Lambda^{12} \mathrm{C}$ obtained using the $\left(\mathrm{K}^{-}, \pi^{-}\right)$reaction at the CERN proton synchrotron (ref. 7).

with a neutron-hole character. The situation is just what one might expect to get from transforming a neutron in the nucleus to a $\Lambda$.

In Figure 13 are shown excitation spectra for $\Lambda^{13} \mathrm{C}$ for $(K, \pi)$ reaction angles of $0^{\circ}$ and $15^{\circ}$. The complexity of these spectra as compared to the $\Lambda^{12} \mathrm{C}$ excitation is obvious from the figure. The angular distributions of the five major peaks of Figure 13 are shown in Figure 14, and the suggested particle hole configurations are listed in Table 1.

Table I

\begin{tabular}{|c|c|c|c|}
\hline Peak $\#$ & Excitation Energy & $\begin{array}{l}\text { Core Energy and } \\
\text { (spin-isospin) }\end{array}$ & $\begin{array}{l}\text { Configuration } \\
\left(\mathrm{n}^{-1}, \Lambda\right)\end{array}$ \\
\hline $\begin{array}{l}1 \\
2 \\
3 \\
4 \\
5\end{array}$ & $\begin{array}{l}0 \\
4.4 \\
10.4 \\
16.4 \\
25.7 \\
25.7 \\
25.7\end{array}$ & $\begin{array}{r}0\left(0^{+}, 0\right) \\
4.43\left(2^{+}, 0\right) \\
0\left(0^{+}, 0\right) \\
4.43\left(2^{+}, 0\right) \\
12.71\left(1^{+}, 0\right) \\
15.11\left(1^{+}, 1\right) \\
16.11\left(2^{+}, 1\right)\end{array}$ & $\begin{array}{ll}\left(\mathrm{P}_{1 / 2},\right. & \left.\mathrm{s}_{1 / 2}\right) \\
\left(\mathrm{p}_{3 / 2},\right. & \left.\mathrm{s}_{1 / 2}\right) \\
\left(\mathrm{p}_{1 / 2},\right. & \left.\mathrm{s}_{1 / 2}, 3 / 2\right) \\
\left(\mathrm{p}_{3 / 2},\right. & \left.\mathrm{p}_{1 / 2}, 3 / 2\right) \\
\left(\mathrm{p}_{3 / 2},\right. & \left.\mathrm{p}_{1 / 2}, 3 / 2\right) \\
\left(\mathrm{p}_{3} / 2,\right. & \left.\mathrm{p}_{1 / 2}, 3 / 2\right) \\
\left(\mathrm{p}_{3 / 2},\right. & \left.\mathrm{P}_{1 / 2}, 3 / 2\right)\end{array}$ \\
\hline
\end{tabular}



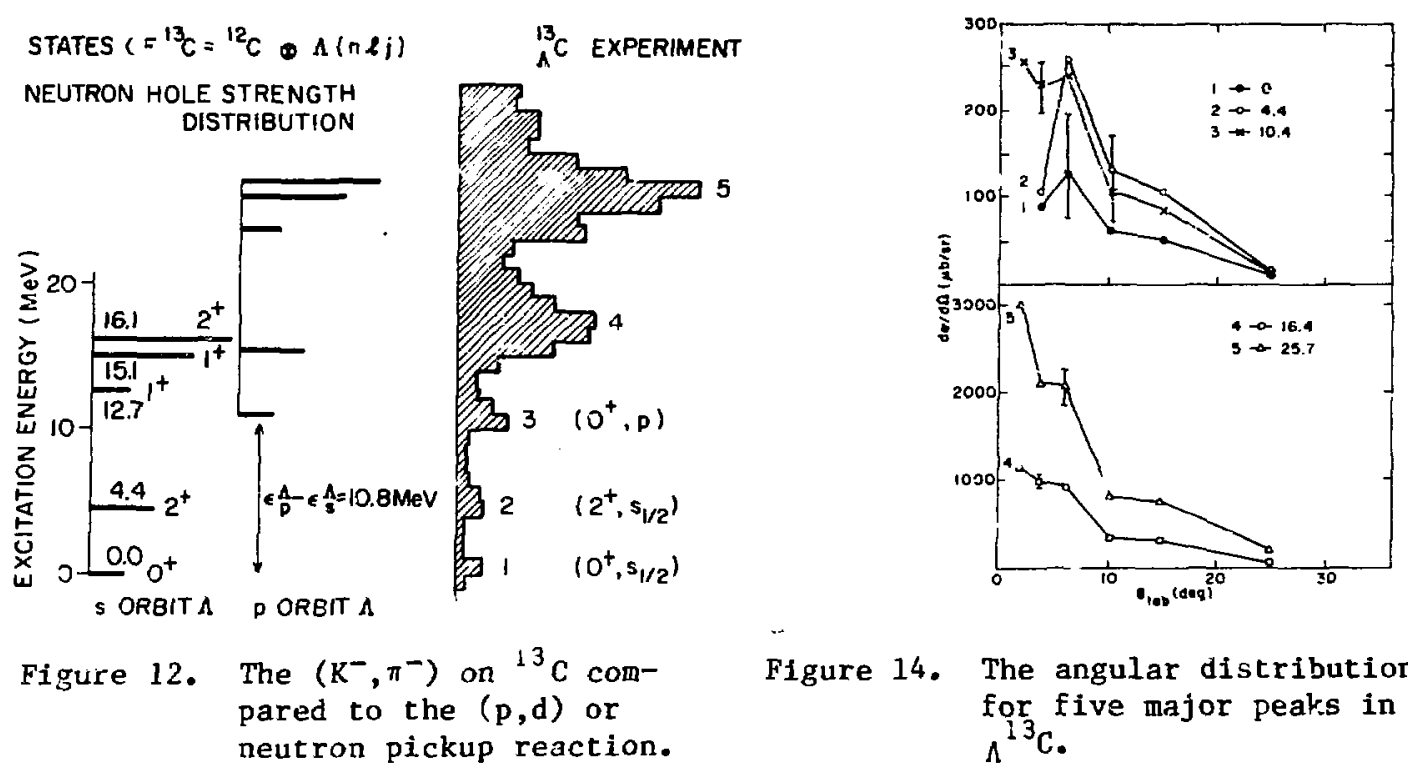

Figure 14. The angular distributions for five major peaks in $\Lambda^{13} \mathrm{C}$.
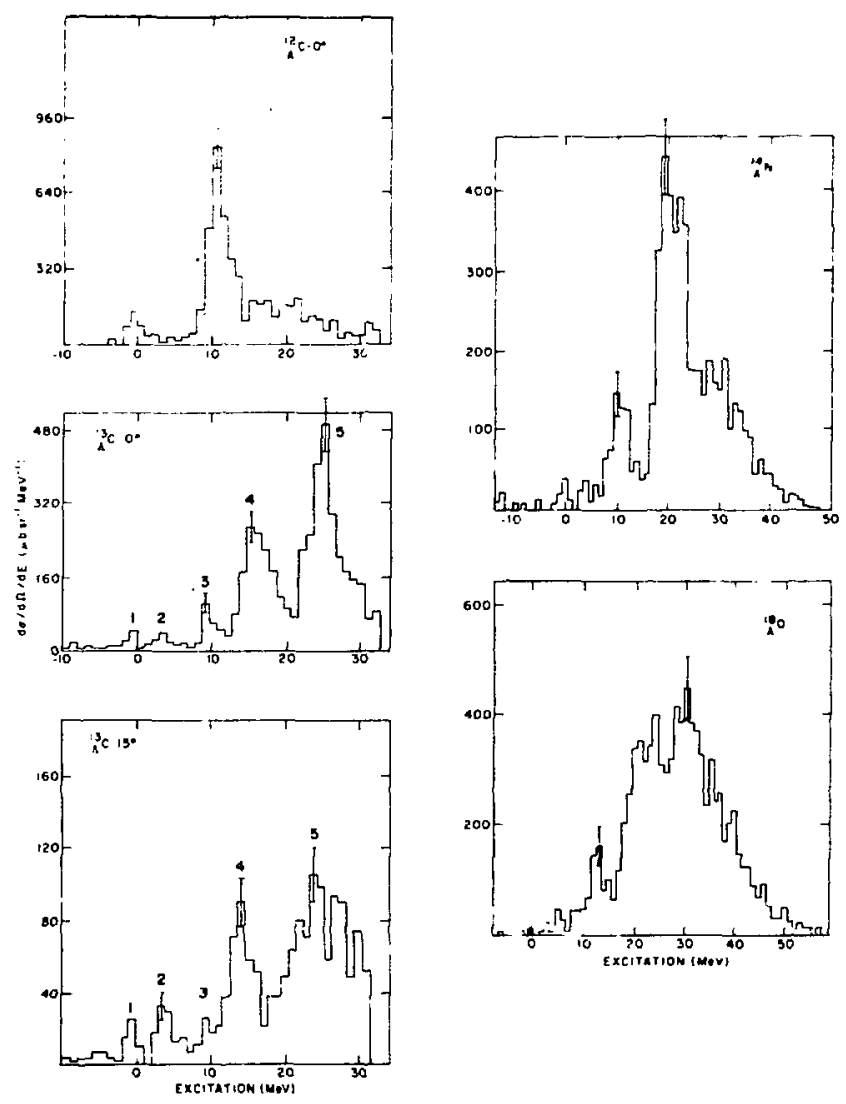

Figure 13. The excitation spectra for $\Lambda^{12} \mathrm{C}$ and $\Lambda^{13} \mathrm{C}$ at $0^{\circ}$ and $15^{\circ}$. 
The interest in this reaction is due, however, not to its similarities to neutron pickup but to its differences from it. If the differences were not manifest, nothing ne would be learned by using kaons instead of protons.

A comprehensive shell-model description for $\Lambda^{13} \mathrm{C}$ has been developed by the Brookhaven Theory Group. ${ }^{8}$ The formalism allows one to deal with the very revealing departures from the simple and weak coupling picture. Furthermore, the analysis allows derivation of information about the $\Lambda$-nucleon effective interaction in the $\mathrm{p}$ shell.

The interesting features concern mainly the $10-\mathrm{MeV}$ and $16-\mathrm{MeV}$ peaks ( 3 and 4 , respectively) and can be summarized as follows.

The $10-\mathrm{MeV}$ peak undergoes very little shift in position in going from $0^{\circ}$ to $15^{\circ}$. This peak at $0^{\circ}$ is understood as the $\mathrm{P} 1 / 2 \Lambda$ coupled to the zerospin ${ }^{12} \mathrm{C}$ core; at $15^{\circ}$, since $\Delta \mathrm{L} \neq 0$ transitions dominate, it must be comprised of a $\mathrm{P}_{3 / 2} \Lambda$ coupled to the ${ }^{12} \mathrm{C}$ gs ccre. The shift in position,

$$
\Delta \mathrm{E}\left(\mathrm{p}_{1 / 2}-\mathrm{p}_{3 / 2}\right)=0.36 \pm 0.3 \mathrm{MeV} \text {, }
$$

is a direct measure of the $\Lambda$-nucleus spin-orbit splitilng. Since the states are coupled to a $0^{+}$core, there is no perturbation by the $\Lambda$-nucleon residual interaction. Hence this is the most direct evidence, so far, inr a weak $\Lambda$-nucleus spin-orbit potential.

On the other hand, the spacing between these peaks at $0^{\circ}$ ( $6 \mathrm{MeV}$ ) differs markedly from the weak-coupling prediction of $4.4 \mathrm{MeV}$. This can be made consistent with the weak spin-orbit effect only by invoking a small $\Lambda$-nucleon effective interaction. Indeed, ignoring the effective interaction gives

$$
\Delta E\left(P_{1} / 2-P_{3} / 2\right)=-1.6 \pm 0.4 \mathrm{MeV}
$$

in sharp disagrecment with the other observation.

Peak 4 also undergoes a downward shift of $1.7 \mathrm{MeV}$ from $0^{\circ}$ to $15^{\circ}$. Again, ignoring any residual interaction would yield

$$
\Delta E\left(p_{1} / 2-p_{3} / 2\right)=-2.2 \pm C .5 \mathrm{MeV}
$$

also a sharp disagreement.

Finally, the relative sizes of peaks 3 and 4 at $0^{\circ} \sigma(3) / \sigma(4)$ disagrees strongly with what is expected from the pickup reaction. The observed ratio of 5 contrasts with the experimental pickup cross-section ratio of 1.5 from the $(p, d)$ reaction on ${ }^{13} \mathrm{c}$.

These discrepancies have been resolved by the BNL Theory Group. ${ }^{9}$ The strength of the quadrupole-quadrupole effective interaction is given in terms of the Slater integral $\mathrm{F}^{(2)}$, where

$$
\mathrm{v}_{\text {eff }}=\mathrm{F}^{(2)} \mathrm{Q}_{\mathrm{r}} \cdot \mathrm{Q}_{\mathrm{N}} \cdot
$$

An adequate fit to all peak positions and intensity ratios can be given for values of $F^{(2)}$ :

$$
-3.4<F^{(2)}<-3.0 \text { and } \Delta E\left(p_{1 / 2}-p_{3 / 2}\right)=0.5 \mathrm{MeV} \text {. }
$$

The residual interaction of $-3 \mathrm{MeV}$ is "weak" compared with the nuclear $1 \mathrm{p}$ shell case where $F(2) \approx-10 \mathrm{MeV}$. Therefore, the intermediate-coupling calculations involving $\Lambda$ 's would be expected to work even better than their nuclear counterparts. 
There is strong evidence that the spin-dependent interactions of the $\Lambda$. with nuclei seem to be small and perhaps not measurable by present experi-mental resolutions involving magnetic analysis of the $(K, \pi)$ reactions. How can better results be achieved to pin down these interactions? One can proceed in analogy again with the nuclear case. There the quite modest resolutions of early spectrometers were complemented by the introduction of highresolution detection of electromagnetic transitions between nuclear levels. Thus the search for hypernuclear $\gamma$ rays, if successful, would provide a precise new spectroscopic tool. In fact, the feasibility of detection of $\gamma$-ray transitions in coincidences with the $\left(\mathrm{K}^{-}, \pi^{-}\right)$reaction has been demonstrated.

Previously observed $\gamma$ transitions from $h$ rernuclei are shown in Figure 15. These experiments ${ }^{9}$ were performed at CERN with stopped kaons on be and $L i$ targets. In these cases, the momentum transfer is high and the hyperfragment is produced in a highly excited state, well above the particle emission threshold. These $\gamma$ rays were seen in coincidence with pions, presumably arising from $\Lambda$ decay. The lines at 1.04 and $1.15 \mathrm{MeV}$ were assigned to the hypernuclei $\Lambda{ }^{4} \mathrm{H}$ and $\Lambda$ He, since there are no nuclear $\gamma$ rays known at $A=7$ or below with these energies. These $\gamma$ rays have been assumed to arise from a spin-flip transition in which the $\Lambda$ spin orientation with respect to the nuclear core undergoes a $180^{\circ}$ reversal.

The $\gamma$ rays from hypernuclei result from particle-stable states which lie below the energy required for emission of either a nucleon or a $\Lambda$ particle; for example, in $\Lambda^{7} \mathrm{Li}$, particle breakup occurs at $3.94 \mathrm{MeV}$,

$$
\Lambda^{7} \mathrm{Li}(3.94 \mathrm{MeV})+{ }^{2} \mathrm{H}+\Lambda^{5} \mathrm{He} \text {, }
$$

and any level of $\Lambda^{7} \mathrm{Li}$ below that energy will qualify as a possible $\gamma$-emitting state.

The lowest energy levels of $\mathrm{p}-\mathrm{shell}$ nuclei will be discussed here, the configurations of interest being

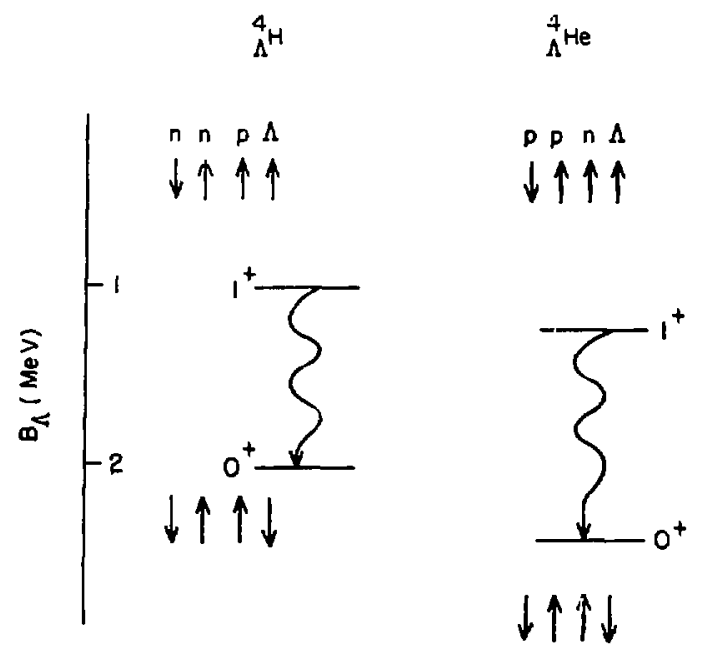

Figure 15. Hypernuclear $Y$ rays in the $A=4$ nuclei, $\Lambda^{4}$ He and $\Lambda^{4} H$. 


$$
\left(S_{N}^{4}\right)\left(P_{N}\right)^{A-5}
$$

and the discussion will be restricted to $0^{\circ}$ hypernuclear formation. Two types of transitions are available:

1) $\gamma$ transitions among the nuclear core levels, the $\Lambda$ playing the role of a spectator only;

2) M-1 spin-flip transitions in the $\Lambda$-nucleus system.

Weisskopf's single-particle estimates indicate that, for energies above about $0.5 \mathrm{MeV}, \mathrm{M} 1$ and E2 transition rates are such that they will dominate weak decay; in special cases, however, hypernuclear isomerism may exist.

The search for hypernuclear $\gamma$ rays was carried out with the BNL hypernuclear spectrometer. The $\gamma$ rays from the target were detected in coincidence with the $\left(\mathrm{K}^{-}, \pi^{-}\right)$trigger in an array of six 5-in.x3-in. and two 8-in.x6-in. NaI detectors, with an overall solid angle of about $30 \%$ of $4 \pi$. For each of the targets studied about $10^{10}$ kaons on target were collected.

It should be emphasized that the production cross sections for these states at forward angles are quite low, in the range of a few tens of unicrobarns/steradian. For experimental success it was necessary to use thick targets, $\approx 6 \mathrm{~g} / \mathrm{cm}^{2}$. The region of interesting levels in $\Lambda^{7} \mathrm{Li}$ is in the boundstate region extending from the gs to an excitation energy of about $3.9 \mathrm{MeV}$.

Dalitz and $\mathrm{Gal}^{11}$ have made extensive calculations of positions and rates of electromagnetic transitions in p-shell hypernuclei, using a weak-coupling model. They have analyzed the separation of hypernuclear doublets in terms of $S_{\Lambda}$, the $\Lambda$-nucleon spin orbit interaction, $\Delta$, the spinmspin interaction, and $T$, a tensor interaction. $\Lambda^{7} \mathrm{Li}$ is a hypernucleus that has been analyzed by Dalitz and Gal. The level scheme they calculated is shown in Figure 16. Re1ativa froduction rates were calculated from the fractional parentage coefficients of Cohen and Kurath. ${ }^{12}$ The splitting of the multiplets built on the $1^{+}$ gs and $3^{+}$first excited state of ${ }^{\delta} \mathrm{Li}$ includes spin-spin and spin-orbit forces, as shown.

The data from the experiment are also shown in Figure 16. At the upper right the $(K, \pi)$ spectrum is shown, divided into various excitation energy cuts, 1 to 4 (Figure 16a). Spectra from those cuts are displayed at the left. Region 1 is background, being the region below the gs in the excitation spectrum; region $2(-8$ to -2$)$ is the bound-state region. A clear peak is seen at $2.04 \mathrm{MeV}$, which is very near the nuclear energy level difference of the $3^{+}$ and $1^{+}$states in ${ }^{6} \mathrm{Li}(2.19 \mathrm{MeV})$. In region $3(-2$ to $4 \mathrm{MeV})$ little or nothing is seen; however, above $4 \mathrm{MeV}$ ( 4 to $10 \mathrm{MeV}$ ) the $3.50^{+}+1^{+}$nuclear $\gamma$-ray line corresponding to the $\gamma$ decay of the first $T=1$ state of $6_{\mathrm{LI}}$ is seen, ${ }_{3}$ indeed it should be.

It is interesting that although the $2-\mathrm{MeV}$ line is quite definitely seen in the $\Lambda^{7} \mathrm{Li}$ case, the $\gamma$ transition is not seen in the corresponding case of ${ }^{6}$ Li. This transition does not compete against decay of the Li into an a particle and a deuteron. The presence of the $\Lambda$ serves to stabilize the system, raising the deuteron emission threshold well above the energy of the $3^{+}$state. Note the extremely small number of events in the $r$-ray spectra. The "2-MeV" peak contains less than 100 counts in the full escape peak for about $2 \times 10^{10}$ kaons incident in target--an efficiency of the order of $10^{-8}$ !

The cuts can be extended further, into higher excitation energies and well above all particle thresholds. Regions 6 and 7 (Figure 16b) are above the thresholds for breakup into $A=4$ plus $A=3$ systems. The appearance of $a$ line at $1.10 \mathrm{MeV}$ supports the CERN data. ${ }^{8}$ The observed value of $1.10 \mathrm{MeV}$ lies near the mean of the 1.04 and 1.15 lines reported. The presence of this peak 


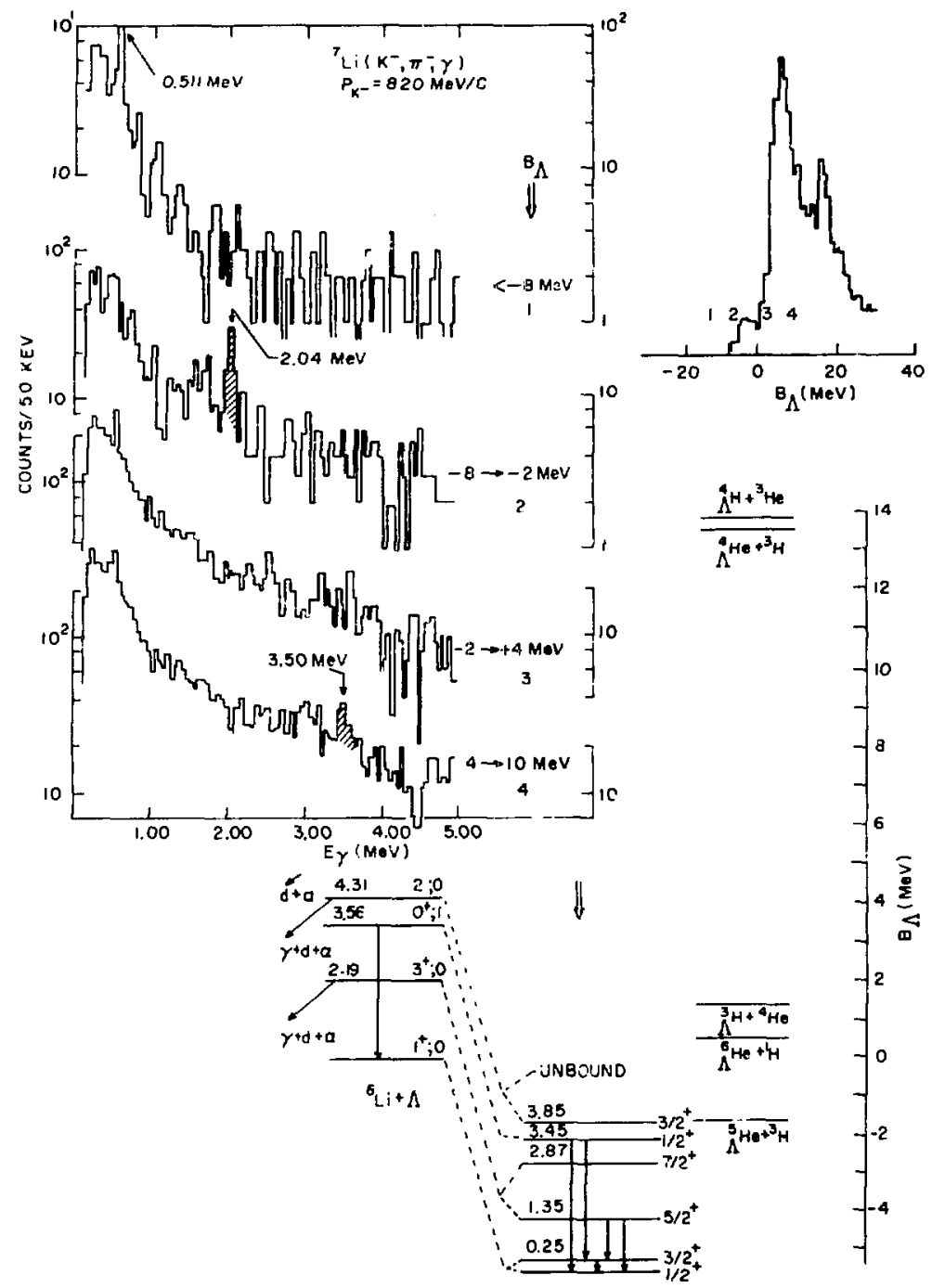

Figure 16a. Level scheme and $\gamma$ spectra for $\Lambda^{\prime}$ Li.

in the proper region of hypernucleus excitation provides strong support for their interpretation. Similar data have been obtained for $\Lambda^{9} \mathrm{Be}$ (Figure 17). In this case the parent state has $0^{+}$for the gs doublet; the $2^{+}$parent is, however, split into a $5 / 2^{+}-3 / 2^{+}$doublet, the upper member of which lies above the threshold for particle stability. As expected, the $\mathrm{BNL}$ data for ${ }^{\mathrm{Be}}$ show only one transition, at $E=3.05 \mathrm{MeV}$. Again, as in $\Lambda^{7} \mathrm{Li}$, the transition energy is very near the core nucleus level difference of $2.94 \mathrm{MeV}$. The line appears in the appropriate excitation energy cut $(-11$ to $1 \mathrm{MeV})$. 


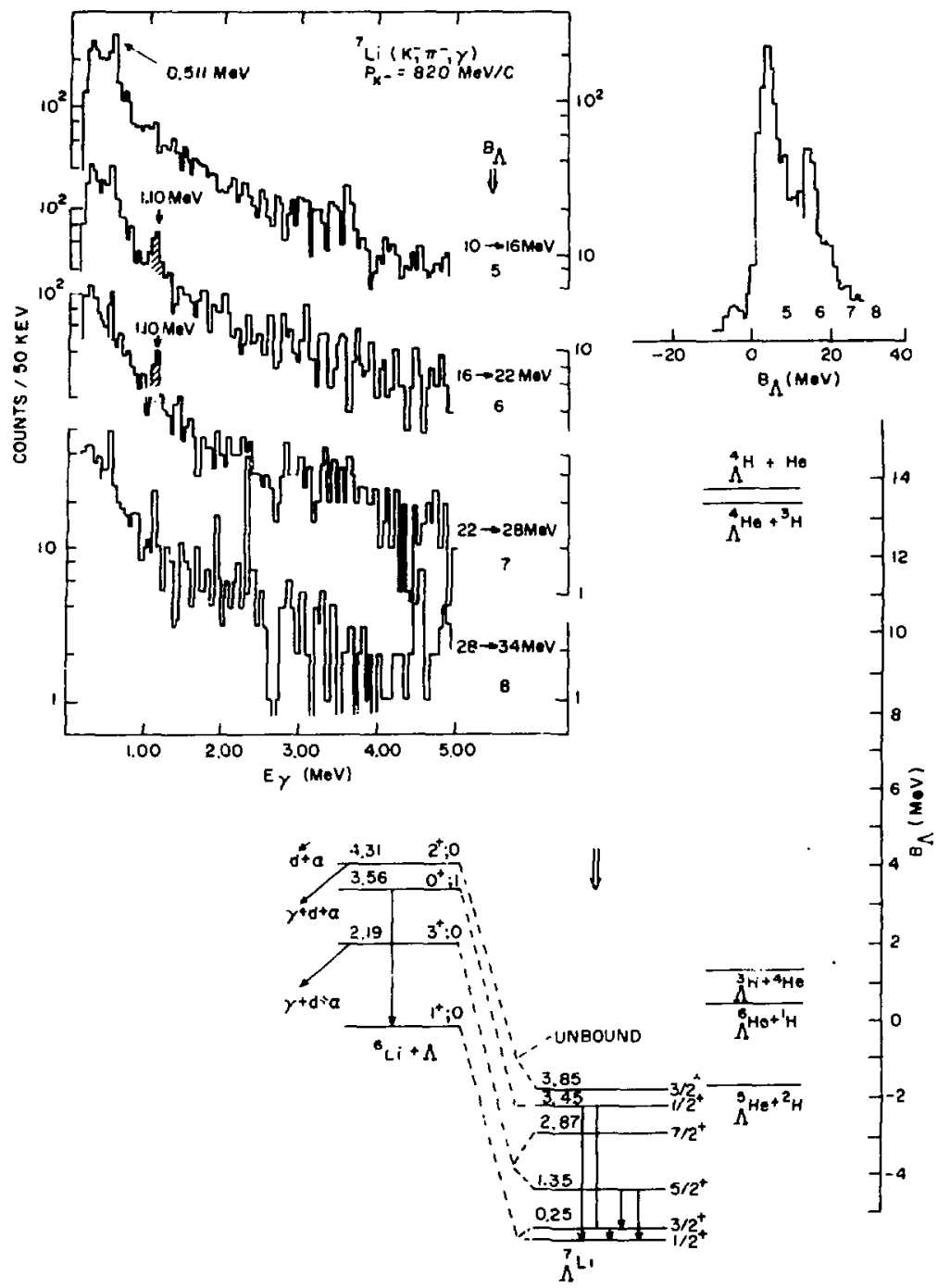

Figure 16b. Level scheme and $\gamma$ spectra for $\Lambda^{\prime} \mathrm{Li}$.

\section{Summary of Results}

The results of these studies can be summarized as follows: In each case the experiments have resulted in detection of the core transition in which the $\Lambda$ plays the role of a spectator and failure to detect the spin flip Ml transition between the two possible $\Lambda$ couplings. This suggests, but does not absolutely require, that the $\Lambda$ spin interactions are small in $p$ shell hypernuclei. The ambiguity is due to there being several terms in the expression for the splitting--spin-soin, spin-orbit, and tensor--so that accidencal 
cancellations are possible. From the BNL data, an upper 11mit of about 200 keV can be placed on the multiplet splittings.

For the future, two more $\gamma$-ray measurements are planned. One is to look for the ground state splitting in $\wedge^{16} 0$ below about $200 \mathrm{keV}$. This can be done by using Ge detectors, whose superior resolution will allow better discrimination against the coincident continuum background, coming mostly from $\pi$ decays. In the range of about $50 \mathrm{keV}, \gamma$ emission competes successfully against the weak decay process.

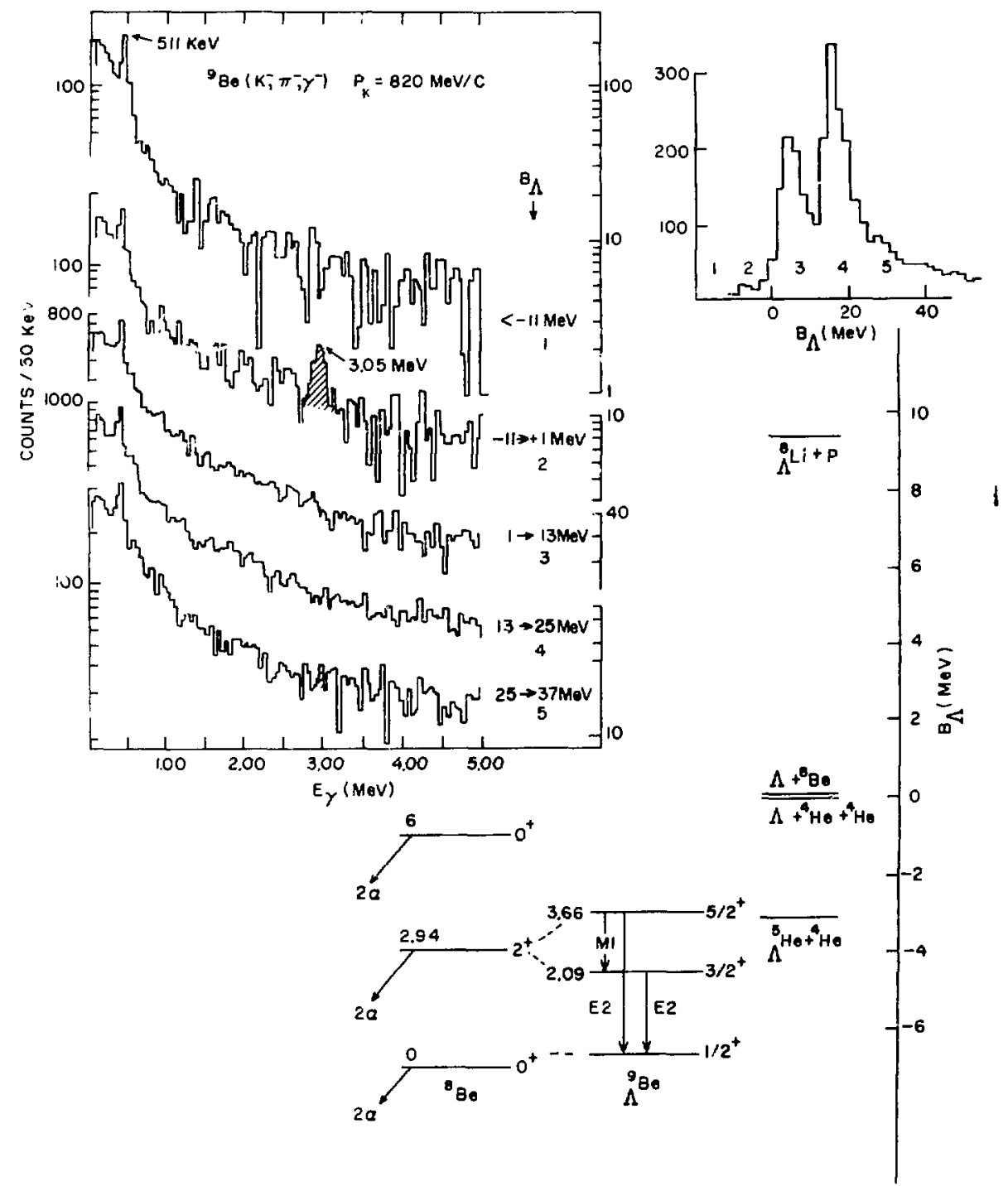

Figure 17. Level scheme and $Y$ spectra for $\Lambda^{9} \mathrm{Be}$. 
The other proposed measurement is to examine the case of the $\Lambda^{13} \mathrm{Cu}$ $\mathrm{P}_{1 / 2}$ substitutional state. If the $\gamma$ ray which deexcites this state to the gs of $\Lambda^{13} \mathrm{C}, \mathrm{E}_{\gamma} \approx 11 \mathrm{MeV}$, can be measured at $0^{\circ}$, and the corresponding P3/2 component measured at $15^{\circ}$, the $0^{\circ}$ to $15^{\circ}$ shift in $\gamma$-ray energy gives directly the $\mathrm{p}_{3 / 2}-\mathrm{p}_{1 / 2}$ splitting. This can probably be done to an accuracy of 30 to $40 \mathrm{keV}$, an order of magnitude better than the present limit.

The spin-dependent $\Lambda$ interactions will ultimately be explainable in terms of the quark structure of these baryons. Pirner ${ }^{13}$ has already suggested that, because of the presence of the strange quark in the $\Lambda$, the simple quark picture predicts a zero spin-orbit interaction. Other authors have used a relativistic mean field theory in which the smallness of the spin-orbit potential arises from the smallness of the $\Lambda \Lambda \sigma$ and $\Lambda \Lambda \omega$ coupling constants in comparison with the iNNo and NNw constants ( $\sigma$ and $\omega$ represent the scalar-isoscalar and vector-isnscalar exchange fields). It is still an open question whether the $\Sigma$ hyperon, which is also known to narticipate in the formation of narrow hypernuclear states, has a small or large spin-orbit potential, or even whether it is of the same sign as in the nuclear case.

Ultimately a coherent theory will be able to treat these problems. That theory awafts the execution of further experiments aimed at establising these splittings, for both $\Lambda$ and $\Sigma$ hypernuclei. Such experiments are now severely restricted by the available kaon flux from accelerators which produce the kasns as secondary particles from proton induced reactions. Proposals have been made for electron accelerators that can produce kanns by associated production, and for "kaon factories" that produce kaons from proton currents of $\approx 100 \mu \mathrm{A}$ at $20 \mathrm{GeV}$. Such new accelerators will provide the tools needed for a detailed study of hypernuclei and thus give further insights into the effective interaction of strange baryons in nuclei.

\section{References}

1. V. F. Weisskopf in Proceedings of the International Conference on Nuclear Structure, Kingston, Canada, p. 903, University of Toronto Press, Toronto, 1960.

2. M. Danysz and J. Pniewski, Phil. Mag. 44, 348 (1953).

3. C. F. Powe11, P. H. Fowler, and D. H. Perkins, The Study of Elementary Particles by the Photographic Method, p. 389, Pergamon Press, London, 1959.

4. V. L. Tele di Sci. Am. 206, 50 (1962).

5. B. Povh, Rep. Prog. Phys. 39, 823 (1976).

6. R. E. Chrien et al., Phys. Lett. 89B, 31 (1979).

7. W. Bruckner et al., Phys. Lett. 73 B, 157 (1978); see also A. Bouyssy, Phys. Lett. 84B, 41 (1979).

8. M. May et al., Phys. Rev. Lett. 47, 1106 (1981).

9. E. H. Auerbach et al., Phys. Rev. Lett. 47, 1110 (1981).

10. H. Piekarz, Proceedings of the International Conference on Hypernuclear and Kaon Physics, p. 73, Heldelberg, Max-Planck-Institut fur Kernphysik, MPIH-1982-V 20, 1982.

11. R. H. Dalitz and A. Gal, Ann. Phys. (N.Y.) 116, 167 (1978).

12. S. Cohen and D. Kurath, Nucl. Phys. 73, 1 (1965); ibid. Al01, 1 (1967).

13. H. J. Pirner, Phys. Lett. 85B, 190 (1979). 